\title{
Signal Processing for Digital Image Enhancement Considering APL in PDP
}

\author{
Soo-Wook Jang ${ }^{1}$, Se-Jin $\mathrm{Pyo}^{2}$, Eun-Su Kim ${ }^{1}$, \\ Sung-Hak Lee ${ }^{1}$, and Kyu-Ik Sohng ${ }^{1}$ \\ 1 School of Electronic Engineering and Computer Science, \\ Kyungpook National University, \\ 1370, Sankyug-Dong, Buk-Gu, Daegu, 702-701, Korea \\ \{jjang, saeloum, shark2, kisohng\}@ee.knu.ac.kr \\ 2 Digital Media R\&D Center, Samsung Electronics Co., LTD., \\ 416, Maetan-3Dong, Yeongtong-Gu, Suwon, Gyeonggi-Do, 443-742, Korea \\ sejin.pyo@samsung.com
}

\begin{abstract}
In this paper, a method for improvement of image quality using the error diffusion which considers the APL process is proposed. In the proposed method, the APL process is performed before the error diffusion process. Simulation results showed that the proposed method has better performances for resolution in images and CCT uniformity according to each grayscale than the conventional method.
\end{abstract}

\section{Introduction}

Plasma display panel (PDP), which is researched actively in recent years, is new flat display technique that replaces flat CRT and TFT-LCD. It is attracting attention as new display device because it has merits that are absolute flat, the maximum of space efficiency, wide sight angle, high luminance and facility of a large display size. In spite of these merits, it has problems such as consumption of high power, high cost and degradation of image quality. The luminance of CRT is controlled by the amount of electro beam adjusted by current. On the other hand, the luminance of panel can't be controlled by amount of current in PDP due to uniformity of light discharged from a phosphor. So the number of sustain pulse is adjusted by desired luminance of panel for constant time. The integral effect or after image effect is used: human perceives the brightness of light by integrating the amount of light generated by sustain pulses for one field. The addressing time is needed to decide discharge of the PDP cell. And it is impossible to control the address electrode every gray-level 1 within one field time in this current PDP driving method. Therefore, the sub-field is used to display 256 gray-levels. One field is separated into several sub-fields and the on or off of each sub-field is controlled.

In current PDP, the major image processing consists of inverse gamma correction, error-diffusion and average picture level (APL). The quantization error, a decrease of number of the gray-scale, occurs during the inverse gamma correction. The digital halftoning method is used to solve this problem and it gives an 
effect which looks like more gray-levels is used as only few gray-levels. Now the most popular method of the digital halftoning is the error-diffusion. Although the error-diffusion is used to distribute this quantization error into the neighboring pixels, the worm-like pattern is inevitably produced [1],[2]. And the worm-like pattern is increased after APL which prevents the excessive temperature of panel and enhances the luminance efficiency of PDP.

However, there are some disadvantages and one of them is the image quality degradation, which is dependent on the digital signal processing. Although image quality of PDP is improving by many researches and experimentations, it still isn't as good as that of CRTs because of various factors. One of them is wormlike pattern generated by an error diffusion process. And the worm-like pattern is severely increased after an average picture level (APL) process. An increased worm-like pattern occur a drop of resolution in image and a change of correlated color temperature (CCT) according to each grayscale.

In this paper, we propose new method for digital image enhancement considering the APL in PDP. In the proposed method, the APL process is performed before the error diffusion process. Simulation results showed that the proposed method has better performances for resolution in images and CCT uniformity according to each grayscale than the conventional method.

\section{Image Processing in the Current PDP}

In current PDP, the major image processing consists of inverse gamma correction, error-diffusion and APL. Fig. 1 shows the block diagram of image processing in the current PDP. The inverse gamma correction is needed to display the gamma-corrected image, because PDP has nearly linear characteristic about output luminance for input gray-level. Digital input data of 8 bits is transformed into the data of bits more than 8 bits in inverse gamma correction processing. Generally, the relationship of input gray-level and output luminance in the image display device is shown in Eq. (1). In this Eq., $i$ is the digital input gray-level, $\gamma$ is display gamma value and $\mathrm{k}$ is constant for normalization.

$$
Y(i)=k \cdot\left(\frac{i}{255}\right)^{\gamma}
$$

The error-diffusion of various digital halftoning methods is used to distribute the errors which occur during the preprocessor. It is mainly used to reduce the quantization error produced by the inverse gamma correction. The difference between the ideal output value of image and the actual output value of it is considered as error in this method. Generally, if the number of output data bits is increase, the quantization error produced by the inverse gamma correction is reduced. And the APL processing is mainly used to prevent the excessive temperature of panel and enhances the luminance efficiency of PDP. The APL maintains a uniform average level of the picture signal during active scanning time integrated over a frame period.

Even though the numerous digital halftoning techniques had been developed in the last twenty years, the one that has emerged as a standard because of its 


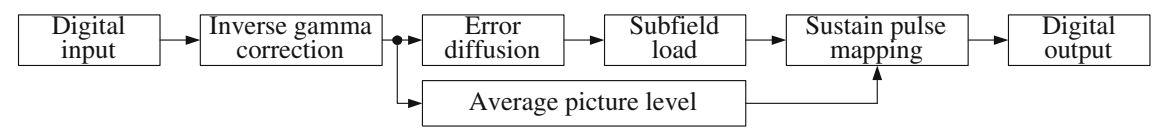

Fig. 1. Block diagram of signal processing in current PDP

simplicity and quality of output is the so-called error-diffusion algorithm [2]-[4]. This algorithm, first proposed by Floyd and Steinberg [5], is schematically shown in Fig. 2. The error-diffusion is used to solve the problem about the degradation of grayscale resolution in the dark area. The error-diffusion can be considered as a feedback system with two major operations - quantization and filtering, as illustrated in Fig. 2. The thresholding operation determines the values of output halftone. The filtering operation is usually implemented by diffusion the weighted binarization error to the anti-causal nearest neighbors. The weights are specified by the filter [6]. The difference between the pixel's ideal output digital value and the actual output digital value is considered as error in this method. To achieve the effect of continuous-tone illusion, this error is distributed to the four neighboring pixels that have not been processed yet, according to the matrix proposed by Floyd and Steinberg [5]. Despite widespread usage in many applications, worm-like patterns at some tone levels and limit-cycle behavior in mid-tones and quartertones are two major artifacts associated with Floyd and Steinberg error-diffusion. This worm-like pattern reduces especially the image quality of the PDP, because it is increased by the next processing like the APL. Also, it is easily detected by human's visual perception, because the most of current PDP is the standard definition panel and the size of one pixel is large relatively.

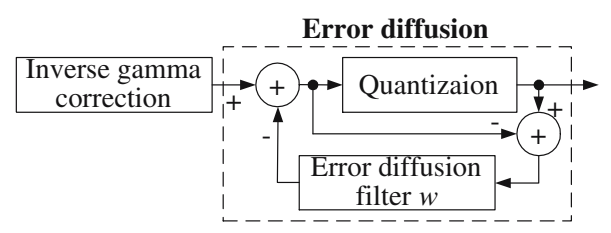

Fig. 2. Error diffusion algorithm

\section{Proposed Method for Digital Image Enhancement Considering APL}

Fig. 3 shows the flow chart of proposed method to improve the digital image quality considering the APL. The proposed method performs all image processing before the error-diffusion processing. 8 bits digital input data are stored with 12 bits after the inverse gamma correction and it is transformed into 16 bits data according to average level of the picture signal during active scanning time integrated over a frame period. The 11 bits data of 16 bits is the integer part and 


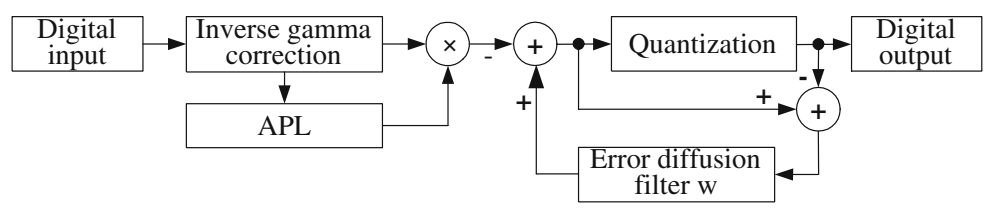

Fig. 3. Flow chart of proposed signal processing with error diffusion

the 5 bits data of it is fraction part for the error-diffusion. Finally, 10 bits data is used to load the subfield memory. The each processing of the proposed method to improve the digital image quality considering the APL is illustrated closely in the next paragraph.

CRT, which is widely used as image display device until now, has nonlinear characteristic about output luminance for input gray-level. On the other hand, PDP has nearly linear characteristic about output luminance for input gray-level. Therefore, inverse gamma correction is needed to display the gamma-corrected image. When the inverse gamma correction is applied, the difference between the target luminance and actual output luminance is produced because the number of grayscale which can be actually displayed in PDP is decreased specially in the dark area. In PDP, we define inverse gamma correction Eq. (2) to show luminance characteristic like the CRT's.

$$
R(r)=R_{\text {white }} \cdot\left(\frac{r}{255}\right)^{\gamma}, G(g)=G_{\text {white }} \cdot\left(\frac{g}{255}\right)^{\gamma}, B(b)=B_{\text {white }} \cdot\left(\frac{b}{255}\right)^{\gamma}
$$

The $r, g$, and $b$ are the digital input values of each color channel and the $R_{w h i t e}, G_{w h i t e}$, and $B_{w h i t e}$ are white level values of each color channel. The results of Eq. (2) show the digital output values for digital input values $r, g$, and $b$. Although it is computed to a fraction, it can be displayed with only integer values. Therefore, ideally computed fraction values have to be transformed into integer values. Table 1 shows the number of grayscale as output data bits in inverse gamma correction processing. It illustrates that the number of grayscale is 255 when the number of output data bit is infinity. In the proposed method to improve the digital image quality considering the APL, the 12 bits are used to minimize a decrease of the number of grayscale generated by inverse gamma correction processing and consider the design of hardware. The degradation of the grayscale resolution is produced in the low gray-levels despite an increase of the number of output data bit.

The great variety of image processing methods using digital halftoning has been suggested to improve this grayscale resolution in the low gray-levels. The

Table 1. The number of grayscale as output bits in inverse gamma corrention

\begin{tabular}{|c|c|c|c|c|c|}
\hline The numver of output bit & 8 & 9 & 10 & 11 & 12 \\
\hline The numver of output bit & 183 & 215 & 233 & 243 & 248 \\
\hline
\end{tabular}


digital half-toning is the process of transforming images with only a few discrete tone levels into the continuous-tone images.

\section{Experiments and Results}

A comparison of simulated images using the conventional and proposed signal processing method is shown in Fig. 4. The simulated images are one which videogamma correction is processed, because it is displayed on standard CRT. The proposed PDP signal processing method has improved performance especially in aspect of increase of the number of grayscale and reduction of distributed error. The PSNR of the proposed method are $7.2 \mathrm{~dB}$ higher than that of the conventional method at gray scale image, and the PSNR of the proposed method are $6.9 \mathrm{~dB}$ higher than that of the conventional method at color image respectively.

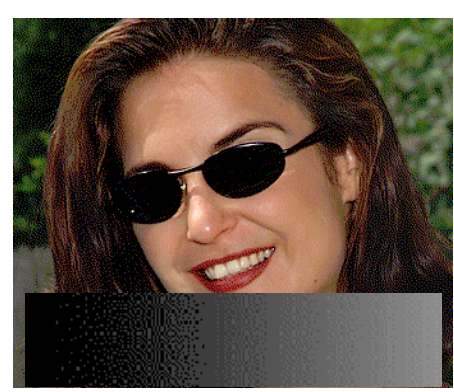

(a)

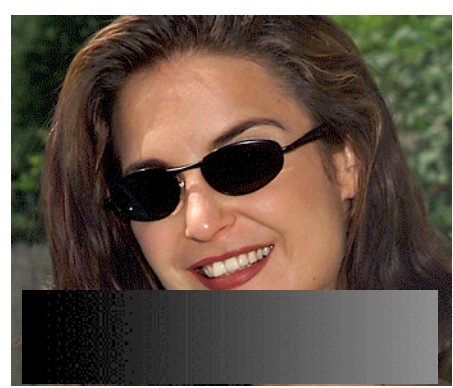

(b)

Fig. 4. A comparison of simulated images: (a) Conventional and (b) proposed signal processing method

The proposed signal processing method enables to display the luminance with one step of the sustain pulse and prevents the increase of the worm-like patterns generated by error-diffusion. Table 2 shows the number of grayscale for the number of sustain pulse in the several the APL, namely, the maximum number of sustain pulse. These illustrate that the luminance of panel is controlled by one step of the sustain pulse and the digital image quality is improved by preventing the increase of the worm-like patterns generated by error-diffusion.

Table 2. The number of grayscale for the number of sustain pulse in the several APL

\begin{tabular}{|c|c|c|}
\hline \multirow{2}{*}{ Maximum numver of sustain pulse } & \multicolumn{2}{|c|}{ The number of grayscale } \\
\cline { 2 - 3 } & Conventional & Proposed \\
\hline 200 & 207 & 222 \\
\hline 255 & 230 & 230 \\
\hline 510 & 230 & 242 \\
\hline 1020 & 230 & 249 \\
\hline
\end{tabular}




\section{Conclusions}

APL processing method is useful technique which prevents the excessive temperature of panel and enhances the luminance efficiency of PDP by maintaining a uniform average voltage of the picture signal during active scanning time integrated over a frame period. In the signal processing method of current PDP, although the error-diffusion is used to distribute this error into the neighboring pixels, the worm-like pattern is inevitably produced. And the worm-like pattern is increased after APL. So the proposed signal processing method enables to display the luminance in one step of the sustain pulse and prevents the increase of the worm-like patterns. In conclusion, the proposed signal processing method gives the excellent effectiveness for improve-ment of the digital image quality considering the APL processing for PDP.

\section{References}

[1] Li, P., Allebath, J. P.: Tone-dependent error diffusion, IEEE Transactions on Image Processing, Vol. 13, (2004) 201-215.

[2] Ulichney, R.: Digital Halftoning, MIT Press, Cambridge, Mass., (1987).

[3] Yoichi Sato, Kimio Amemiya, and Masataka Uchidoi: Recent Progresses of Device Per-formance and Picture Quality in Color Plasma Displays, IDW'00 Digest, (2000) 695-698.

[4] Peter R. Jones: Evolution of halftoning technology in the United States patent literature, Journal of Electronic Imaging, Vol. 3, No. 3, (1994) 257-275.

[5] Robert W. Floyd and Louis Steinberg: An Adaptive Algorithm for Spatial Grayscale, Proceedings of the Society for Information Display, Vol. 17, No. 2, (1976) 75-77.

[6] Ti-chiun Chang and Jan P. Allebach: Memory Efficient Error Diffusion, IEEE Transactions on image processing, Vol. 12, No. 11, (2003) 1352-1366. 